\title{
Confined Friedel oscillations on Au(111) terraces probed by thermovoltage scanning tunneling microscopy
}

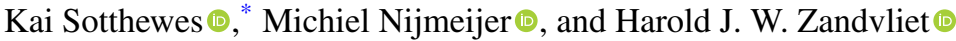 \\ Physics of Interfaces and Nanomaterials, MESA+ Institute for Nanotechnology, University of Twente, \\ P.O. Box 217, 7500 AE Enschede, The Netherlands
}

(Received 29 January 2021; revised 7 June 2021; accepted 11 June 2021; published 29 June 2021)

\begin{abstract}
The spatial dependence of the local density of states at the Fermi level of a stepped $\mathrm{Au}(111)$ surface is studied by thermovoltage scanning tunneling microscopy. The periodicity of the standing waves is not given by $\lambda_{\mathrm{F}} / 2=$ $1.8 \mathrm{~nm}$, as expected based on the band diagram of $\mathrm{Au}(111)$, but rather varies between 1.5 and $2.1 \mathrm{~nm}$ depending on the exact width of the terraces. This counterintuitive result can be understood by considering the superposition of incident and reflected electron waves, which have a periodicity of $\lambda_{\mathrm{F}} / 2$. The change in periodicity is a direct consequence of the decaying nature of the Friedel oscillations.
\end{abstract}

DOI: 10.1103/PhysRevB.103.245311

\section{INTRODUCTION}

Standing waves are ubiquitous in optical, electronic, and mechanical experiments. On the nanoscale, electron standing wave patterns have been extensively studied by scanning tunneling microscopy (STM) since the seminal work of Crommie et al. $[1,2]$. These authors used the STM to drag individual iron atoms on a copper substrate to a predefined position. One of the nanostructures they assembled via atomic manipulation was a circle consisting of 48 iron atoms. This circle of iron atoms, also referred to as a quantum corral, acts as a barrier for the surface state electrons at the Fermi level. As the incoming and outgoing electron waves interfere with each other, a circular standing wave pattern develops inside the quantum corral. This electron density modulation of the surface is referred to as a Friedel oscillation and is given by

$$
\rho_{\mathrm{s}}(r, E)=\rho_{\mathrm{b}}+C \frac{\cos \left(2 k_{\mathrm{F}}|r|+\delta\right)}{|r|^{D}},
$$

where $k_{\mathrm{F}}$ is the Fermi wave vector, $\rho_{\mathrm{b}}$ the bulk contribution to the electron density, $\delta$ a phase factor, $D$ the dimensionality of the electron system, and $|r|$ the distance from the scattering center.

There are various ways to measure Friedel oscillations with STM. For metals it is usually sufficient to make a topographic map at a small sample bias, as in this case only the electrons in the vicinity of the Fermi level contribute to the tunnel current [1-3]. In order to measure the dispersion relation of the surface state electrons, the differential conductivity $(d I / d V)$, which is proportional to the local density of states (LDOS), is measured as a function of the bias voltage $(V)$ [4-9]. Alternatively, one can make a spatial map of the thermovoltage $\left(V_{\text {th }}\right)$ that arises if a temperature gradient is applied across the scanning tunneling microscopy junction. The thermovoltage, which is proportional to the derivative of the LDOS to the energy, provides direct information on the spatial variation of the Friedel oscillations [10-13].

*Corresponding author: k.sotthewes@utwente.nl
To date the vast majority of scanning tunneling microscopy studies have been focused on electron standing wave patterns near defects or steps on noble metal surfaces that possess a two-dimensional surface state, such as $\mathrm{Au}(111), \mathrm{Ag}(111)$, and $\mathrm{Cu}(111)[1-6,12,14-18]$. During the last few years, however, the technique has also been applied to more diverse materials, such as semiconductors [19], topological insulators [20], twodimensional materials [21], and ferroelectric materials [9].

The confinement of surface state electrons on terraces owing to the presence of step edges has been studied in quite some detail $[4,6-8,22]$. The standing wave patterns are well described by the Fabry-Pérot resonator model for terrace widths $L$ in the range of $\lambda_{\mathrm{F}} / 2$ to $3 \lambda_{\mathrm{F}} / 2$, where $\lambda_{\mathrm{F}}$ is the Fermi wavelength $[4,6] . \lambda_{F}$ can be determined from the dispersion of the $\mathrm{Au}(111)$ surface state and is equal to $\lambda_{\mathrm{F}}=3.6 \mathrm{~nm}$. For terrace widths smaller than $\lambda_{\mathrm{F}} / 2$, the confinement changes from terrace modulation to step modulation [7,22].

So far, for terrace widths exceeding $\lambda_{\mathrm{F}} / 2$, a systematic study of the interference pattern as a function of the terrace width is still lacking. In this paper, we probe the interference pattern for electrons confined on terraces with $L>3 \lambda_{\mathrm{F}} / 2$ using thermovoltage STM. Surprisingly, the measured wavelength of the standing waves is altered and no longer equal to $\lambda_{\mathrm{F}} / 2$. The confinement influences the observed oscillations for terrace widths $L>3 \lambda_{\mathrm{F}} / 2$. We will show that at these length scales the decaying nature of the Friedel oscillations plays a pivotal role. The oscillations in this regime are formed by the interference of several waves formed by the superposition of incident and reflected electron waves at the step edges of the $\mathrm{Au}(111)$ surface. The waves have a wave vector equal to $k_{\mathrm{F}}$, similar as for an infinitely large terrace, but the wave vector of the resulting interference pattern varies depending on the exact terrace width.

\section{EXPERIMENTAL DETAILS}

The experiments are performed with a RHK Technology UHV3000 variable temperature STM equipped with R9 hardware and a home-built thermovoltage module [13]. The tip is 
kept at room temperature $\left(T_{\mathrm{T}}=293 \mathrm{~K}\right)$, while the sample is cooled down to $T_{\mathrm{S}}=220 \mathrm{~K}$. During the thermovoltage measurement, the tip-sample distance $(\Delta z)$ is reduced by $0.3 \mathrm{~nm}$ with respect to the set point used in the topographic measurement. The sample used in these experiments consists of a 200-nm-thick $\mathrm{Au}$ (111) layer on mica purchased from Phasis (Geneva, Switzerland).

\section{RESULTS AND DISCUSSION}

A thermovoltage arises between the tip and surface if a temperature gradient is applied across the STM tunnel junction. $V_{\text {th }}$ is the voltage needed to compensate for the thermally generated tunneling current. The thermally generated tunneling current is caused by inequalities in the Fermi-Dirac distributions, as well as differences in the LDOS at the Fermi levels of tip and sample, and has two opposite contributions with energies above and below the Fermi level. Støvneng and Lipavský [23] derived the following expression for $V_{\text {th }}$ within the Tersoff-Hamann [24] approximation:

$$
V_{\mathrm{th}}=\frac{\pi^{2} k_{\mathrm{B}}^{2} T_{\mathrm{m}} \Delta T}{3 e}\left[\frac{1}{\rho_{\mathrm{t}}} \frac{\partial \rho_{\mathrm{t}}}{\partial E}+\frac{1}{\rho_{\mathrm{s}}} \frac{\partial \rho_{\mathrm{s}}}{\partial E}+\frac{z}{\hbar} \sqrt{\frac{2 m_{0}}{\phi}}\right],
$$

where $k_{\mathrm{B}}$ is the Boltzmann constant, $\hbar$ is the reduced Planck constant, $m_{0}$ is the electron mass, $e$ is the electron charge, $\phi$ is the work function, $z$ is the tip-sample distance, and $\rho_{\mathrm{t}}$ and $\rho_{\mathrm{s}}$ are the local density of states of the tip and sample, respectively. $T_{\mathrm{m}}=\frac{1}{2}\left(T_{\mathrm{t}}+T_{\mathrm{s}}\right)$ is the mean temperature with the tip $\left(T_{\mathrm{t}}\right)$ and sample $\left(T_{\mathrm{s}}\right)$ temperature and $\Delta T=T_{\mathrm{t}}-T_{\mathrm{s}}$ the temperature difference. Since $\rho_{\mathrm{t}}$ and $z$ are assumed to be constant, the dominant term for the spatial variation of $V_{\text {th }}$ is given by $\frac{1}{\rho_{\mathrm{s}}} \frac{\partial \rho_{\mathrm{s}}}{\partial E}$. The latter results in a high sensitivity in the LDOS modulations around $E_{\mathrm{F}}$.

The LDOS $\rho_{\mathrm{s}}(r, E)$ of electrons with wave vector $k(E)$ scattered at a monatomic step can be calculated by summing up all states (surface and bulk states) at energy $E$ [1],

$$
\rho_{\mathrm{s}}(x, E)=\rho_{\mathrm{b}}(E)+L_{0}\left\{1-R J_{0}[2 k(E) x]\right\},
$$

where $L_{0}=m^{*} /\left(\pi \hbar^{2}\right), x$ the distance from the step, $\rho_{\mathrm{b}}(E)$ the bulk LDOS (assumed to be independent of $x$ and $y$ ), $R$ the reflectivity of the step $[R=0.4$ for $\mathrm{Au}(111)][12,25], k^{2}=$ $2 m^{*} E / \hbar^{2}$, and $J_{0}$ the zero-order Bessel function. Note here, that for the thermovoltage measurements, the energy is equal to the Fermi energy and therefore constant and hence $\rho_{\mathrm{b}}(E)$ is a constant and $k(E)$ is equal to $k_{\mathrm{F}}$.

Equation (3) is only valid in the regime where the LDOS is linear at the Fermi level, which is only in the direct vicinity of the step edge. Therefore Eq. (2) is no longer applicable because the LDOS is no longer linear around $E_{\mathrm{F}}$ [26]. A spatial dependent expression is derived in Ref. [26] for $V_{\mathrm{th}}(x)$ based on the following approximations [26,27]: (i) $e V_{\text {th }} \ll k_{\mathrm{B}} T_{\mathrm{t}, \mathrm{s}}$, (ii) $4 k_{\mathrm{B}} T_{\mathrm{t}, \mathrm{s}} \partial \rho_{\mathrm{s}} / \partial E \ll \rho_{\mathrm{s}}$, and (iii) $4 k_{\mathrm{B}} T_{\mathrm{t}, \mathrm{s}} \ll \varepsilon_{0}$ and is given by

$$
\begin{gathered}
V_{\mathrm{th}}(x)=\frac{C J_{1}\left(2 k_{\mathrm{F}} x\right) h_{\mathrm{Z}}(x)}{1-C J_{0}\left(2 k_{\mathrm{F}} x\right) h_{\mathrm{N}}(x)}, \\
h_{\mathrm{Z}}(x)=\frac{\varepsilon_{0}}{e k_{\mathrm{F}}}\left(\frac{s_{\mathrm{s}}}{\sinh \left(s_{\mathrm{s}} x\right)}-\frac{s_{\mathrm{t}}}{\sinh \left(s_{\mathrm{t}} x\right)}\right) ; \\
h_{\mathrm{N}}(x)=\frac{1}{2}\left(\frac{s_{\mathrm{s}} x}{\sinh \left(s_{\mathrm{s}} x\right)}+\frac{s_{\mathrm{t}} x}{\sinh \left(s_{\mathrm{t}} x\right)}\right)
\end{gathered}
$$
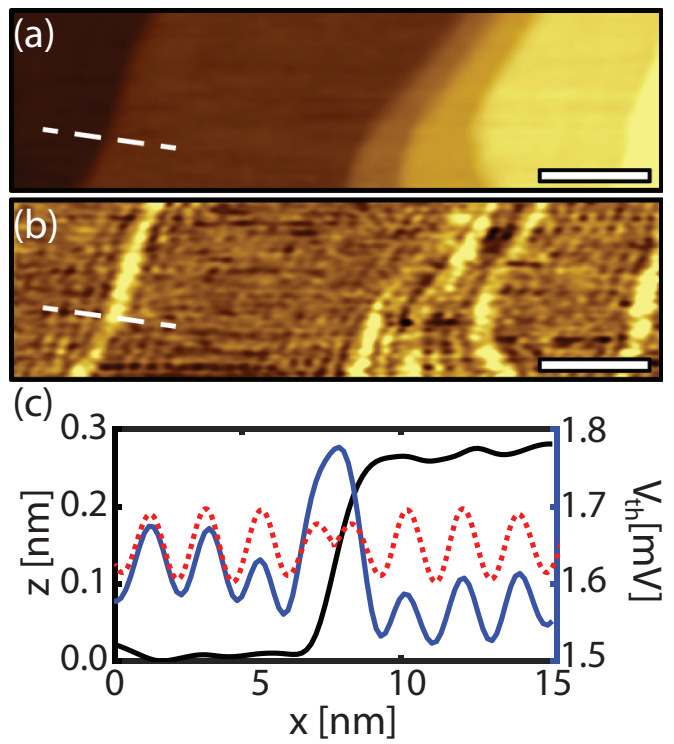

FIG. 1. (a) Topography image $\left(75 \times 21 \mathrm{~nm}^{2}\right.$, scale bar is $\left.13 \mathrm{~nm}\right)$ of $\mathrm{Au}(111)$ showing a stepped $\mathrm{Au}(111)$ surface. The tunneling parameters are $300 \mathrm{mV}$ and $300 \mathrm{pA}$. (b) The corresponding thermovoltage map for $T_{\mathrm{T}}=293 \mathrm{~K}, T_{\mathrm{S}}=220 \mathrm{~K}$, and $\Delta z=0.3 \mathrm{~nm}$, showing surface electron interference patterns. (c) The height (left, black) and $V_{\text {th }}$ (right, blue) profile measured simultaneously along the dashed line in (a) and (b). The red dashed line is the model described by Eq. (4), using $k_{\mathrm{F}}=1.74 \mathrm{~nm}^{-1}$.

with $C=P R=0.2 . P$ is the surface state contribution with respect to the bulk and is experimentally determined to be $P=0.5[1,28] . s_{\mathrm{s}}=\pi k_{\mathrm{B}} T_{\mathrm{s}} k_{\mathrm{F}} / \varepsilon_{0}$ and $s_{\mathrm{t}}=\pi k_{\mathrm{B}} T_{\mathrm{t}} k_{\mathrm{F}} / \varepsilon_{0}$ with $\varepsilon_{0}$ the energy minimum of the surface state $(-0.41 \mathrm{eV}$ for $\operatorname{Au}(111)[29,30])$.

Figure 1 shows the topography and the simultaneously obtained thermovoltage map of the $\mathrm{Au}(111)$ surface. In the thermovoltage map [see Fig. 1(b)] the step edges show up as $200 \mu \mathrm{V}$ high peaks with respect to the average thermovoltage value of the terrace. The increase in the thermovoltage at the step is a result of the enhanced coupling of the tunneling electrons with the bulk states [10,11]. In the vicinity of step edges, the thermovoltage shows a spatially decaying oscillating behavior, which can be attributed to electronic standing waves. These Friedel oscillations have a periodicity of $1.8 \mathrm{~nm}$ [see Fig. 1(c)]. Since no bias is applied, we are dealing with Fermi electrons. Using the dispersion relation of the surface state of $\mathrm{Au}(111)[29,30]$,

$$
E=\varepsilon_{0}+\frac{\hbar^{2} k^{2}}{2 m^{*}}
$$

with $\varepsilon_{0}=-0.41 \mathrm{eV}$ and $m^{*}=0.28$, we find a corresponding wave vector of $1.74 \mathrm{~nm}^{-1}\left(\lambda_{\mathrm{F}}=3.6 \mathrm{~nm}\right)$. In good agreement with the experimental observations and previous studies [3], this leads to a periodicity of $1.8 \mathrm{~nm}\left(\lambda_{\mathrm{F}} / 2\right)$ in the LDOS at $E_{\mathrm{F}}$.

The red line in Fig. 1(c) is the model as described by Eq. (4) and qualitatively fits the measured Friedel oscillations. In order to fit the model, the effective boundary location is taken close to the midpoint of the rise in the topological scan and at the maximum of the thermovoltage measurement [5]. The 

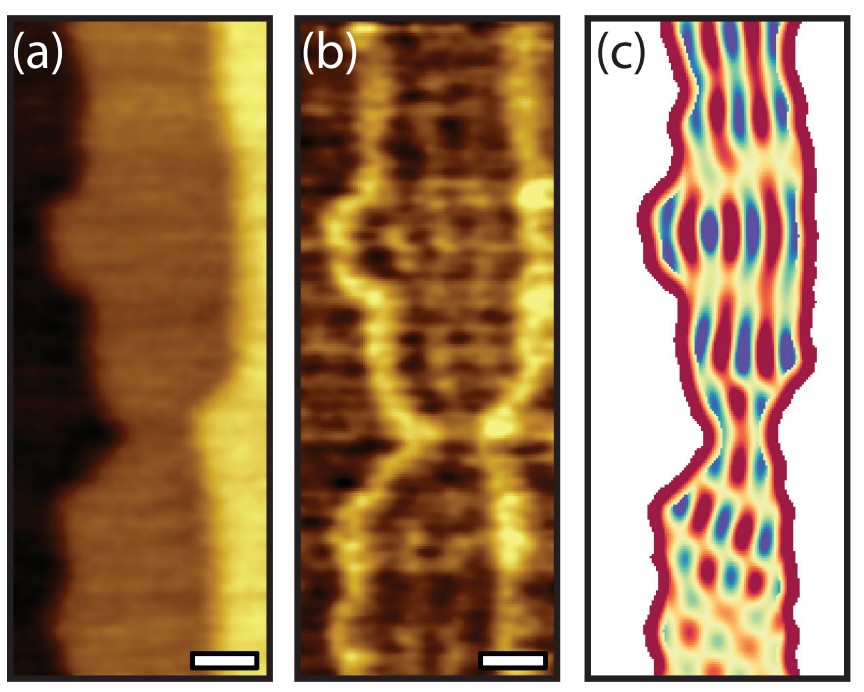

FIG. 2. (a) Topography image $\left(30 \times 12 \mathrm{~nm}^{2}\right.$, scale bar $\left.3 \mathrm{~nm}\right)$ of $\mathrm{Au}(111)$ showing a stepped $\mathrm{Au}(111)$ surface. The tunneling parameters are $300 \mathrm{mV}$ and $300 \mathrm{pA}$. (b) The simultaneously recorded thermovoltage map for $T_{\mathrm{T}}=293 \mathrm{~K}, T_{\mathrm{S}}=220 \mathrm{~K}$, and $\Delta z=0.3 \mathrm{~nm}$, showing surface electron interference patterns confined between the steps. (c) Simulated thermovoltage image based on the interference between two Friedel oscillations. The oscillations are simulated using Eq. (7).

first oscillation is completely covered by the signal originating from the step.

Figure 2(a) shows the topography of a stepped $\mathrm{Au}(111)$ surface. The terrace width varies between 3.5 and $10 \mathrm{~nm}$, which is in the range of $\lambda_{\mathrm{F}}$ to $3 \lambda_{\mathrm{F}}$. In the corresponding thermovoltage map [Fig. 2(b)], the electron standing waves are clearly visible. The number of observed oscillations depends on the terrace width. In Fig. 3(a) the number of peaks $\left(P_{\mathrm{N}}\right)$ and the periodicity $\left(\tilde{\bar{\lambda}}_{\text {in }}=2 \lambda_{\text {in }} / \lambda_{\mathrm{F}}\right.$, where $\lambda_{\text {in }}$ is the measured periodicity) are plotted as a function of the terrace width $(L)$. $P_{\mathrm{N}}$ increases stepwise as a function of $L$. In contrast to the particle-in-a-box model, oscillations exist for every terrace width, except for $L<4 \mathrm{~nm}$. For $L<\lambda_{\mathrm{F}}$ no oscillations are observed in agreement with previous studies due to the conversion from terrace modulation to step modulation $[7,22]$. The width of the plateaus is approximately equal to the $\lambda_{\mathrm{F}} / 2$. The measured wavelength of the oscillations $\left(\lambda_{\text {in }}\right)$ decreases from 2.1 to $1.5 \mathrm{~nm}\left(\widetilde{\lambda}_{\text {in }}\right.$ varies between 1.2 and 0.8$)$ as $L$ increases and is centered around $1.8 \mathrm{~nm}$, which is equal to $\lambda_{\mathrm{F}} / 2$. When $P_{\mathrm{N}}$ increases, the wavelength first rapidly increases to decrease again with increasing terrace width. This is unexpected as $k$ (and thus $\lambda$ ) is determined by the band diagram and the energy at which the measurement is performed [see Eq. (6)]. Similar to a large terrace (as in Fig. 1), the

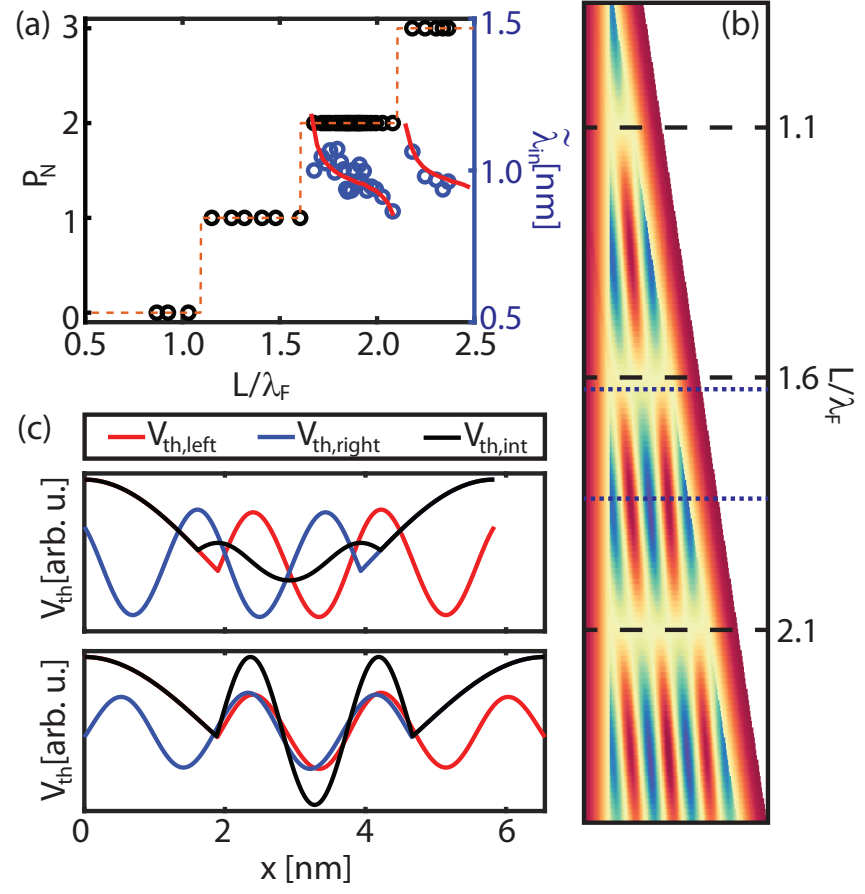

FIG. 3. (a) The number of interference peaks ( $P_{\mathrm{N}}$, left, black) and the peak periodicity $\left(\widetilde{\lambda}_{\text {in }}=\left[2 \lambda_{\text {in }} / \lambda_{F}\right]\right.$, right, blue $)$ as a function of the terrace width $(L)$. The orange $\left(P_{\mathrm{N}}\right)$ and red $\left(\tilde{\lambda}_{i n}\right)$ lines are fits based on the model described by Eq. (7). (b) Simulated thermovoltage map [based on Eq. (7)] for an increasing terrace width $L$. $L$ is varied between 3 and $10 \mathrm{~nm}$. The dashed black lines coincide with the steps in (a). (c) The left (blue line) and right (red line) Friedel oscillation and the resulting interference wave (black line) based on Eq. (7) for (top) $L=5.9 \mathrm{~nm}\left(L / \lambda_{\mathrm{F}}=1.64\right)$ and (bottom) $L=6.5 \mathrm{~nm}\left(L / \lambda_{\mathrm{F}}=1.8\right)$, corresponding to position of the blue dotted line in (b).

wavelength is expected to be equal to $\lambda_{\mathrm{F}} / 2=1.8 \mathrm{~nm}$ with a deviation for terrace widths close to $1.5 \lambda_{\mathrm{F}}$ [7].

The observed interference pattern and the relation between $L, P_{\mathrm{N}}$, and $\lambda_{\text {in }}$ cannot be properly described by the Fabry-Pérot resonator model [4] as oscillations exist for all terrace widths. Therefore, a model is proposed that relies on the interference of decaying standing waves in the LDOS. For electrons in confinement, two steps are involved and therefore several (depending on the number of reflections that are taken into account) wave functions should be considered compared to the two for the single step model [26]. This leads to the following spatial dependent expression for $V_{\mathrm{th}}(x)$ (see the Appendix for the complete derivation):

$$
V_{\mathrm{th}}(x)=\frac{C J_{1}\left(2 k_{\mathrm{F}} x\right) h_{\mathrm{z} 1}(x)+C J_{1}\left[2 k_{\mathrm{F}}(L-x)\right] h_{\mathrm{z} 2}(x)}{1-C J_{0}\left(2 k_{\mathrm{F}} x\right) h_{\mathrm{n} 1}(x)-C J_{0}\left[2 k_{\mathrm{F}}(L-x)\right] h_{\mathrm{n} 2}(x)}
$$

with

$$
\begin{aligned}
& h_{\mathrm{z} 1}(x)=\frac{\varepsilon_{\mathrm{F}}}{e k_{\mathrm{F}}}\left[\frac{s_{\mathrm{s}}}{\sinh \left(s_{\mathrm{s}} x\right)}-\frac{s_{\mathrm{t}}}{\sinh \left(s_{\mathrm{t}} x\right)}\right] ; \quad h_{\mathrm{n} 1}(x)=\frac{1}{2}\left[\frac{s_{\mathrm{t}} x}{\sinh \left(s_{\mathrm{t}} x\right)}+\frac{s_{\mathrm{s}} x}{\sinh \left(s_{\mathrm{s}} x\right)}\right], \\
& h_{\mathrm{z} 2}(x)=\frac{\varepsilon_{\mathrm{F}}}{e k_{\mathrm{F}}}\left[\frac{s_{\mathrm{s}}}{\sinh \left[s_{\mathrm{s}}(L-x)\right]}-\frac{s_{\mathrm{t}}}{\sinh \left[s_{\mathrm{t}}(L-x)\right]}\right] ; \quad h_{\mathrm{n} 2}(x)=\frac{1}{2}\left[\frac{s_{\mathrm{t}}(L-x)}{\sinh \left[s_{\mathrm{t}}(L-x)\right]}+\frac{s_{\mathrm{s}}(L-x)}{\sinh \left[s_{\mathrm{s}}(L-x)\right]}\right]
\end{aligned}
$$


with $s_{\mathrm{s}}=\pi k_{\mathrm{B}} T_{\mathrm{s}} k_{\mathrm{F}} / \varepsilon_{0}$ and $s_{\mathrm{t}}=\pi k_{\mathrm{B}} T_{\mathrm{t}} k_{\mathrm{F}} / \varepsilon_{0}$. Note here that the influence of the step is not taken into account in this model. Although the density of states is given by a linear combination of a single step LDOS starting at $x=0$ [Eq. (3)] and a mirrored step LDOS at $x=L$ (similar to an interference of the LDOS waves), this is not the case for the quantity measured here, i.e., $V_{\text {th }}$. As a result, Eq. (7) deviates from Eq. (4) by the addition of an additional Bessel function in the numerator and denominator.

Figure 2(c) shows the result of the simulation based on the boundaries extracted from Fig. 2(a) and Eq. (7). The waves are generated from point sources located on the boundary [in this case 300, equal to the number of scan lines in Figs. 2(a) and 2(b)] and simulate the Friedel oscillations. The origin of the waves is taken in the midpoint of the rise in the topographical scan [4,5], similar as shown in Fig. 1. The resulting interference pattern is shown in Fig. 2(c). A good agreement is observed between the experiment and the simulation. The influence of the step is added to the existing interference wave in order to fully reproduce the experiment in Fig. 2(b) [31].

In order to explain the observed behavior between $P_{\mathrm{N}}$ and $L$ (as well as $\lambda_{\text {in }}$ and $L$ ), Eq. (7) is used for $3 \mathrm{~nm}<L<10 \mathrm{~nm}$. The result of the model depicted in Fig. 3(b) is plotted in orange $\left(P_{\mathrm{N}}\right)$ and in red $\left(\widetilde{\lambda}_{\text {in }}\right)$ in Fig. 3(a). A similar steplike trend is observed in $P_{\mathrm{N}}$. The plateaus are $\lambda_{\mathrm{F}} / 2$ wide, equivalent to the wavelength of the Friedel oscillations. A visualization of the interference wave as a function of $L$ reveals that the increase in $P_{\mathrm{N}}$ [(Fig. 3(a)] coincides with the appearance of an additional peak [dashed black lines in Fig. 3(b)]. In addition, the observed trend in the apparent wavelength $\left(\lambda_{\text {in }}\right)$ is fully reproduced by the simulation [blue (experimental) and red (theory) lines in Fig. 3(a)]. The general trend is caused by constructive and destructive interference between the decaying waves originating from the two steps. In Fig. 3(c) two interference patterns for different terrace widths are shown. For a terrace width of $5.9 \mathrm{~nm}\left[L / \lambda_{\mathrm{F}}=1.64\right.$, close to a transition point, top panel of Fig. 3(c)] the incoming and outgoing waves are almost fully out of phase resulting in a wave with a relatively large apparent wavelength. The wavelength of the incoming and outgoing waves remains of course equal to $\lambda_{\mathrm{F}}$, as predicted by the band diagram of the $\mathrm{Au}(111)$ surface state, while the wave vector of the interference wave is altered $\left(\lambda_{\text {in }}\right)$. For $L=6.5 \mathrm{~nm}\left[L / \lambda_{\mathrm{F}}=1.8\right.$, bottom panel of Fig. 3(c) $]$ the incoming and outgoing waves are in phase resulting in a wave with a wavelength close to $\lambda_{\mathrm{F}}$.

The observed behavior can only be explained by the superposition of several wave functions. This leads to the interference of decaying standing waves in the LDOS. If nondecaying waves are considered, only energies are allowed in which the wave vector fits the box size, as in the particle-in-a-box model. Therefore, the decaying nature of these oscillations is key for the observed oscillating behavior. The thermovoltage STM provides the possibility to detect the subtle changes in the LDOS due to the strong dependence between $V_{\text {th }}$ and $\frac{1}{\rho_{\mathrm{s}}} \frac{\partial \rho_{\mathrm{s}}}{\partial E}$.

\section{CONCLUSION}

In conclusion, the LDOS of the surface state electrons of $\mathrm{Au}(111)$ is measured between two steps for terrace widths ranging from $\lambda_{F}$ to $3 \lambda_{F}$. Wave patterns with wavelengths ranging from 1.5 to $2.1 \mathrm{~nm}$ are observed, which deviates from the expected $\lambda_{F} / 2$ based on the $\mathrm{Au}(111)$ band diagram. A model is derived based on the interaction between decaying standing waves in the local density of states with a wavelength equal to half of the Fermi wavelength $\left(\lambda_{F} / 2=1.8 \mathrm{~nm}\right)$ within a potential well. The resulting wave pattern exhibits a similar modification in the wavelength as in the experiment. This is a direct result of the decaying nature of the quantum interference waves.

\section{ACKNOWLEDGMENTS}

The authors thank Martin Siekman for technical support and Dr. van Bremen for many fruitful discussions.

\section{APPENDIX}

The surface LDOS for a terrace confined by two step edges can be modeled by considering four wave functions: two incoming waves towards both step edges, and two reflected waves. A random phase $\phi_{R}$ is introduced for the right waves, which describes the random phase of the right waves with respect to the left waves.

$$
\begin{aligned}
\psi_{\mathrm{li}}= & \frac{\psi(z)}{\sqrt{S_{x y}}} \exp \left[-i k_{x}\left(x-x_{1}\right)+i k_{y} y+i \omega t\right], \\
\psi_{\mathrm{lr}}= & \frac{\psi(z)}{\sqrt{S_{x y}}} R \exp \left[i k_{x}\left(x-x_{1}\right)+i k_{y} y+i \omega t+i \phi_{W}\right], \\
\psi_{\mathrm{ri}}= & \frac{\psi(z)}{\sqrt{S_{x y}}} \exp \left[i k_{x}\left(x-x_{2}\right)+i k_{y} y+i \omega t+i \phi_{R}\right], \\
\psi_{\mathrm{rr}}= & \frac{\psi(z)}{\sqrt{S_{x y}}} R \exp \left[-i k_{x}\left(x-x_{2}\right)+i k_{y} y+i \omega t+i \phi_{R}+i \phi_{W}\right] .
\end{aligned}
$$

Resulting in a total wave function of

$$
\begin{aligned}
\psi\left(\vec{r}, \vec{k}_{\|}\right)= & \psi_{\mathrm{li}}+\psi_{\mathrm{lr}}+\psi_{\mathrm{ri}}+\psi_{\mathrm{rr}}=\frac{\psi(z)}{\sqrt{S_{x y}}} \exp \left(i k_{y} y+i \omega t\right)\left\{\exp \left[-i k_{x}\left(x-x_{1}\right)\right]+R \exp \left[i k_{x}(x-x 1)+i \phi_{W}\right]\right. \\
& \left.+\exp \left[i k_{x}\left(x-x_{2}\right)+i \phi_{R}\right]+R \exp \left[-i k_{x}\left(x-x_{2}\right)+i \phi_{R}+i \phi_{W}\right]\right\}
\end{aligned}
$$


such that the LDOS $\rho_{\vec{k}_{\|}}$becomes

$$
\rho_{\vec{k}_{\|}}=\left|\psi\left(\vec{r}, \vec{k}_{\|}\right)\right|^{2}=\frac{|\psi(z)|^{2}}{S_{x y}}\left[2\left(1+R^{2}\right)+2 R \cos \left[2 k_{x}(x-x 1)+\phi_{W}\right]+2 R \cos \left[2 k_{x}\left(x_{2}-x\right)-i \phi_{W}\right]+\sim \cos \left(C \pm \phi_{R}\right)\right] .
$$

To include all existing wave functions on this terrace, the function should first be integrated over all random phase possibilities:

$$
\begin{aligned}
\rho_{\vec{k}_{\|}} & =2 \frac{|\psi(z)|^{2}}{S_{x y}}\left\{1+R^{2}+2 R \cos \left[2 k_{x}(x-x 1)+\phi_{W}\right]+2 R \cos \left[2 k_{x}\left(x_{2}-x\right)-i \phi_{W}\right]\right\}+\sim \frac{1}{2 \pi} \int_{0}^{2 \pi} \cos \left(C \pm \phi_{R}\right) d \phi_{R} \\
& =2 \frac{|\psi(z)|^{2}}{S_{x y}}\left\{1+R^{2}+R \cos \left[2 k_{x}(x-x 1)+\phi_{W}\right]+R \cos \left[2 k_{x}\left(x_{2}-x\right)-i \phi_{W}\right]\right\} .
\end{aligned}
$$

The term $\sim \frac{1}{2 \pi} \int_{0}^{2 \pi} \cos \left(C \pm \phi_{R}\right) d \phi_{R}$ describes multiple cosine functions. Their arguments consist of a variable (independent of $\left.\phi_{R}\right) \pm \phi_{R}$. All of these integrals will result in 0 after evaluation.

To obtain the net electron density, we assume $\phi_{W}=\pi$ and correct for the inflow of electrons at the steps:

$$
\begin{gathered}
\left|\psi_{\text {inflow }}\left(\vec{r}, \vec{k}_{\|}\right)\right|^{2}=2 \frac{|\psi(z)|^{2}}{S_{x y}}\left(1-R^{2}\right), \\
\rho_{\vec{k}_{ \pm \|}}=\left|\psi\left(\vec{r}, \vec{k}_{\|}\right)\right|^{2}+\left|\psi_{\text {inflow }}\left(\vec{r}, \vec{k}_{\|}\right)\right|^{2}=2 \frac{|\psi(z)|^{2}}{S_{x y}}\left\{2-R \cos \left[2 k_{x}(x-x 1)\right]-R \cos \left[2 k_{x}\left(x_{2}-x\right)\right]\right\} .
\end{gathered}
$$

Similarly as for a single step (see Ref. [26]), we must integrate over all possible surface states with a wave vector $k_{\|}$:

$$
\begin{gathered}
\rho_{\text {surf }}(\vec{r}, E)=\frac{m^{*}}{\pi \hbar^{2}} S_{x y} \int_{0}^{\pi} \rho_{\vec{k} \|}(\vec{r}, E) \frac{d \alpha}{2 \pi}, \\
\rho_{\text {surf }}(\vec{r}, E)=\frac{m^{*}}{\pi \hbar^{2}}|\psi(z)|^{2} \frac{1}{\pi} \int_{0}^{\pi}\left\{2-R \cos \left[2 k_{\|}(E)\left(x-x_{1}\right) * \sin (\alpha)\right]-R \cos \left[2 k_{\|}(E)\left(x_{2}-x\right) * \sin (\alpha)\right]\right\} d \alpha, \\
\rho_{\text {surf }}(\vec{r}, E)=\rho_{\text {surf }}(z)\left\{2-R J_{0}\left[2 k_{\|}(E)\left(x-x_{1}\right)\right]-R J_{0}\left[2 k_{\|}(E)\left(x_{2}-x\right)\right]\right\},
\end{gathered}
$$

where the definition of the zeroth-order Bessel function $J_{0}$ is used,

$$
J_{0}\left[2 k_{\|}(E) x\right]=\frac{1}{\pi} \int_{0}^{\pi} \cos \left[2 k_{\|}(E) x \sin (\alpha)\right] d \alpha .
$$

Setting $x_{1}=0$ and $x_{2}=L$ we obtain the surface LDOS dependent on the terrace width $L$ :

$$
\rho_{\text {surf }}(\vec{r}, E)=\rho_{\text {surf }}(z)\left\{2-R J_{0}\left[2 k_{\|}(E) x\right]-R J_{0}\left[2 k_{\|}(E)(L-x)\right]\right\},
$$

which is the same as the linear combination of a single step LDOS at $x=0$ and a mirrored single step LDOS at $x=L$. The derivation for the total thermovoltage signal is then obtained by following the same procedure as in Ref. [26], but replacing the surface electrons LDOS $\rho_{\text {surf }}(\vec{r}, E)$ with Eq. (A8). The result is then

$$
V_{\mathrm{th}}(x)=\frac{C J_{1}\left(2 k_{\mathrm{F}} x\right) h_{\mathrm{z} 1}(x)+C J_{1}\left[2 k_{\mathrm{F}}(L-x)\right] h_{\mathrm{z} 2}(x)}{1-C J_{0}\left(2 k_{\mathrm{F}} x\right) h_{\mathrm{n} 1}(x)-C J_{0}\left[2 k_{\mathrm{F}}(L-x)\right] h_{\mathrm{n} 2}(x)}
$$

with

$$
\begin{gathered}
h_{\mathrm{z} 1}(x)=\frac{\varepsilon_{\mathrm{F}}}{e k_{\mathrm{F}}}\left[\frac{s_{\mathrm{s}}}{\sinh \left(s_{\mathrm{s}} x\right)}-\frac{s_{\mathrm{t}}}{\sinh \left(s_{\mathrm{t}} x\right)}\right] ; \quad h_{\mathrm{n} 1}(x)=\frac{1}{2}\left[\frac{s_{\mathrm{t}} x}{\sinh \left(s_{\mathrm{t}} x\right)}+\frac{s_{\mathrm{s}} x}{\sinh \left(s_{\mathrm{s}} x\right)}\right], \\
h_{\mathrm{z} 2}(x)=\frac{\varepsilon_{\mathrm{F}}}{e k_{\mathrm{F}}}\left[\frac{s_{\mathrm{s}}}{\sinh \left[s_{\mathrm{s}}(L-x)\right]}-\frac{s_{\mathrm{t}}}{\sinh \left[s_{\mathrm{t}}(L-x)\right]}\right] ; \quad h_{\mathrm{n} 2}(x)=\frac{1}{2}\left[\frac{s_{\mathrm{t}}(L-x)}{\sinh \left[s_{\mathrm{t}}(L-x)\right]}+\frac{s_{\mathrm{s}}(L-x)}{\sinh \left[s_{\mathrm{s}}(L-x)\right]}\right] .
\end{gathered}
$$

[1] M. Crommie, C. Lutz, and D. Eigler, Imaging standing waves in a two-dimensional electron gas, Nature (London) $\mathbf{3 6 3}, 524$ (1993).

[2] M. Crommie, C. Lutz, and D. Eigler, Confinement of electrons to quantum corrals on a metal surface, Science 262, 218 (1993).
[3] P. Avouris and I.-W. Lyo, Observation of quantum-size effects at room temperature on metal surfaces with STM, Science 264, 942 (1994).

[4] L. Bürgi, O. Jeandupeux, A. Hirstein, H. Brune, and K. Kern, Confinement of Surface State Electrons in Fabry-Perot Resonators, Phys. Rev. Lett. 81, 5370 (1998). 
[5] J. Li, W.-D. Schneider, R. Berndt, and S. Crampin, Electron Confinement to Nanoscale $\mathrm{Ag}$ Islands on Ag(111): A Quantitative Study, Phys. Rev. Lett. 80, 3332 (1998).

[6] L. Bürgi, O. Jeandupeux, H. Brune, and K. Kern, Probing Hot-Electron Dynamics at Surfaces with a Cold Scanning Tunneling Microscope, Phys. Rev. Lett. 82, 4516 (1999).

[7] K. Morgenstern, K.-F. Braun, and K.-H. Rieder, Surface-State Depopulation on Small Ag(111) Terraces, Phys. Rev. Lett. 89, 226801 (2002).

[8] K. Morgenstern, K.-H. Rieder, and G. A. Fiete, Disorderinduced local-density-of-states oscillations on narrow $\mathrm{Ag}(111)$ terraces, Phys. Rev. B 71, 155413 (2005).

[9] K. Chang, B. J. Miller, H. Yang, H. Lin, S. S. P. Parkin, S. Barraza-Lopez, Q.-K. Xue, X. Chen, and S.-H. Ji, Standing Waves Induced by Valley-Mismatched Domains in Ferroelectric SnTe Monolayers, Phys. Rev. Lett. 122, 206402 (2019).

[10] D. Hoffmann, J. Y. Grand, R. Möller, A. Rettenberger, and K. Läuger, Thermovoltage across a vacuum barrier investigated by scanning tunneling microscopy: Imaging of standing electron waves, Phys. Rev. B 52, 13796 (1995).

[11] A. Schneider, M. Wenderoth, K. Engel, M. Rosentreter, A. Heinrich, and R. Ulbrich, Local electronic structure at steps on $\mathrm{Au}(111)$ investigated by the thermovoltage in scanning tunneling microscopy, Appl. Phys. A 66, S161 (1998).

[12] K. J. Engel, M. Wenderoth, N. Quaas, T. C. G. Reusch, K. Sauthoff, and R. G. Ulbrich, Thermovoltage mapping of standing electron waves on $\mathrm{Au}(111)$ surfaces at low temperatures, Phys. Rev. B 63, 165402 (2001).

[13] K. Sotthewes, M. H. Siekman, and H. J. W. Zandvliet, A method to measure the thermovoltage with a high spatial resolution, Appl. Phys. Lett. 108, 141601 (2016).

[14] E. Heller, M. Crommie, C. Lutz, and D. Eigler, Scattering and absorption of surface electron waves in quantum corrals, Nature (London) 369, 464 (1994).

[15] O. Sánchez, J. M. García, P. Segovia, J. Alvarez, A. L. Vázquez de Parga, J. E. Ortega, M. Prietsch, and R. Miranda, Lateral confinement of surface states on stepped $\mathrm{Cu}(111)$, Phys. Rev. B 52, 7894 (1995).

[16] H. Manoharam, C. Lutz, and D. Eigler, Quantum mirages formed by coherent projection of electronic structure, Nature (London) 403, 512 (2000).

[17] J. Repp, F. Moresco, G. Meyer, K.-H. Rieder, P. Hyldgaard, and M. Persson, Substrate Mediated Long-Range Oscillatory Interaction Between Adatoms: $\mathrm{Cu} / \mathrm{Cu}(111)$, Phys. Rev. Lett. 85, 2981 (2000).
[18] H. Yang, A. J. Mayne, M. Boucherit, G. Comtet, G. Dujardin, and Y. Kuk, Quantum interference channeling at graphene edges, Nano Lett. 10, 943 (2010).

[19] K. Kanisawa, M. J. Butcher, H. Yamaguchi, and Y. Hirayama, Imaging of Friedel Oscillation Patterns of Two-Dimensionally Accumulated Electrons at Epitaxially Grown InAs(111) A Surfaces, Phys. Rev. Lett. 86, 3384 (2001).

[20] J. Wang, W. Li, P. Cheng, C. Song, T. Zhang, P. Deng, X. Chen, X. Ma, K. He, J.-F. Jia, Q.-K. Xue, and B.-F. Zhu, Power-law decay of standing waves on the surface of topological insulators, Phys. Rev. B 84, 235447 (2011).

[21] P. Mallet, I. Brihuega, S. Bose, M. M. Ugeda, J. M. GómezRodríguez, K. Kern, and J. Y. Veuillen, Role of pseudospin in quasiparticle interferences in epitaxial graphene probed by high-resolution scanning tunneling microscopy, Phys. Rev. B 86, 045444 (2012).

[22] J. E. Ortega, S. Speller, A. R. Bachmann, A. Mascaraque, E. G. Michel, A. Närmann, A. Mugarza, A. Rubio, and F. J. Himpsel, Electron Wave Function at a Vicinal Surface: Switch from Terrace to Step Modulation, Phys. Rev. Lett. 84, 6110 (2000).

[23] J. A. Støvneng and P. Lipavský, Thermopower in scanningtunneling-microscope experiments, Phys. Rev. B 42, 9214 (1990).

[24] J. Tersoff and D. R. Hamann, Theory of the scanning tunneling microscope, Phys. Rev. B 31, 805 (1985).

[25] G. Hörmandinger and J. B. Pendry, Interaction of surface states with rows of adsorbed atoms and other one-dimensional scatterers, Phys. Rev. B 50, 18607 (1994).

[26] K. Engel, Raster-Thermospannungs-Mikroskopie der Interferenz von Elektronenwellen auf der $\mathrm{Au}(111)$-Oberfläche, Ph.D. thesis, Universität Göttingen, 2001.

[27] J. Homoth, M. Wenderoth, K. J. Engel, T. Druga, S. Loth, and R. G. Ulbrich, Reconstruction of the local density of states in $\mathrm{Ag}(111)$ surfaces using scanning tunneling potentiometry, Phys. Rev. B 76, 193407 (2007).

[28] W. Chen, V. Madhavan, T. Jamneala, and M. F. Crommie, Scanning Tunneling Microscopy Observation of an Electronic Superlattice at the Surface of Clean Gold, Phys. Rev. Lett. 80, 1469 (1998).

[29] S. D. Kevan and R. H. Gaylord, High-resolution photoemission study of the electronic structure of the noble-metal (111) surfaces, Phys. Rev. B 36, 5809 (1987).

[30] P. Avouris, I.-W. Lyo, R. Walkup, and Y. Hasegawa, Real space imaging of electron scattering phenomena at metal surfaces, J. Vac. Sci. Technol. B 12, 1447 (1994).

[31] See Supplemental Material at http://link.aps.org/supplemental/ 10.1103/PhysRevB.103.245311 for the implementation of the step signal into the simulations. 